IFN- $\gamma$ than wild-type cells. Together with the observation that IL-27 induced the phosphorylation of STAT1 (signal transducer and activator of transcription 1), these results led the authors to suggest that WSX1 signalling is required for STAT1 activation, and subsequent $\mathrm{T}_{\mathrm{H}} 1$-cell differentiation, only when IL-12 is limiting, and that in situations in which IL-12 is plentiful — such as during T. gondii infection - WSX1 is not required for the generation of a $\mathrm{T}_{\mathrm{H}} 1$-cell response and is, in fact, required to prevent overproduction of cytokines.

Using Wsx1-deficient mice these two studies have revealed a new role for WSX1 as a negative regulator of the cytokine response to intracellular pathogens, making it a potential therapeutic target for the treatment of inflammatory diseases.

Karen Honey

(2) References and links ORIGINAL RESEARCH PAPERS Hamano, S. et al. WSX-1 is required for resistance to Trypanosoma cruzi infection by regulation of proinflammatory cytokine production. Immunity 19, 657-667 (2003) Villarino, A. et al. The IL-27R (WSX-1) is required to suppress $T$ cell hyperactivity during infection. Immunity 19, 645-655 (2003)

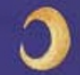

\section{T-CELL MEMORY}

\section{Staying alive with IL-7}

The generation and survival of memory T-cell populations are crucial for prolonged protective immunity following infection. Although the factors involved in $\mathrm{CD}^{+}$memory T-cell homeostasis are well defined, an important role for interleukin-7 (IL-7) in the generation and maintenance of $\mathrm{CD}^{+}$memory $\mathrm{T}$ cells has only recently been clarified by two groups, reporting in The Journal of Experimental Medicine.

Activated effector cells are highly susceptible to apoptosis; factors that rescue cells from programmed cell death or activation-induced cell death are crucial for the transition of effector $\mathrm{T}$ cells to persistent memory cells. The common $\gamma$ chain receptor cytokines IL-7 and IL-15 have previously been shown to be important for the survival of naive $\mathrm{T}$ cells and $\mathrm{CD} 8^{+}$memory $\mathrm{T}$ cells. The survival of naive T cells depends on stimulation through the T-cell receptor (TCR) and is usually accompanied by cell division, whereas the survival of memory populations seems to be independent of TCR ligation and division. Previous in vivo and in vitro studies on the role of IL-7 in the homeostasis of $\mathrm{CD} 4^{+} \mathrm{T}$-cell memory have produced conflicting data.

Both reports start by showing that addition of low doses of IL-7 to CD4 ${ }^{+}$memory cells in vitro promotes cell survival in the absence of cell division. Increased survival was concomitant with the upregulation of expression of the antiapoptotic protein Bcl-2 and memory cell markers. Using different approaches, they then went on to see whether the same was true in vivo.

JiChu Li et al. generated activated $\mathrm{CD}^{+}$ effector T cells in vitro from wild-type and IL-7 receptor (IL-7R)-deficient mice and co-transferred them to wild-type mice or MHC class-II-deficient mice to assess the dependency of effector-memory transition on IL-7 signalling and TCR ligation in vivo. After only two days, the survival of the transferred $I L-7 R^{-/-}$ cells was markedly lower than wild-type cells, which expressed increased levels of Bcl-2. Recovery of transferred cells was also IL-7 dependent in MHC class $\mathrm{II}^{-/-}$hosts.

Kondrack et al.generated resting memory cells in vivo by transferring naive $\mathrm{CD} 4^{+} \mathrm{T}$ cells specific for ovalbumin (OVA)-derived peptide (OT-II cells) to intact or lymphopaenic recipients, which were then immunized with OVA. In-vivo-induced resting memory cells were repurified and transferred to wild-type or $I L-7^{-1-}$ hosts. Few OT-II

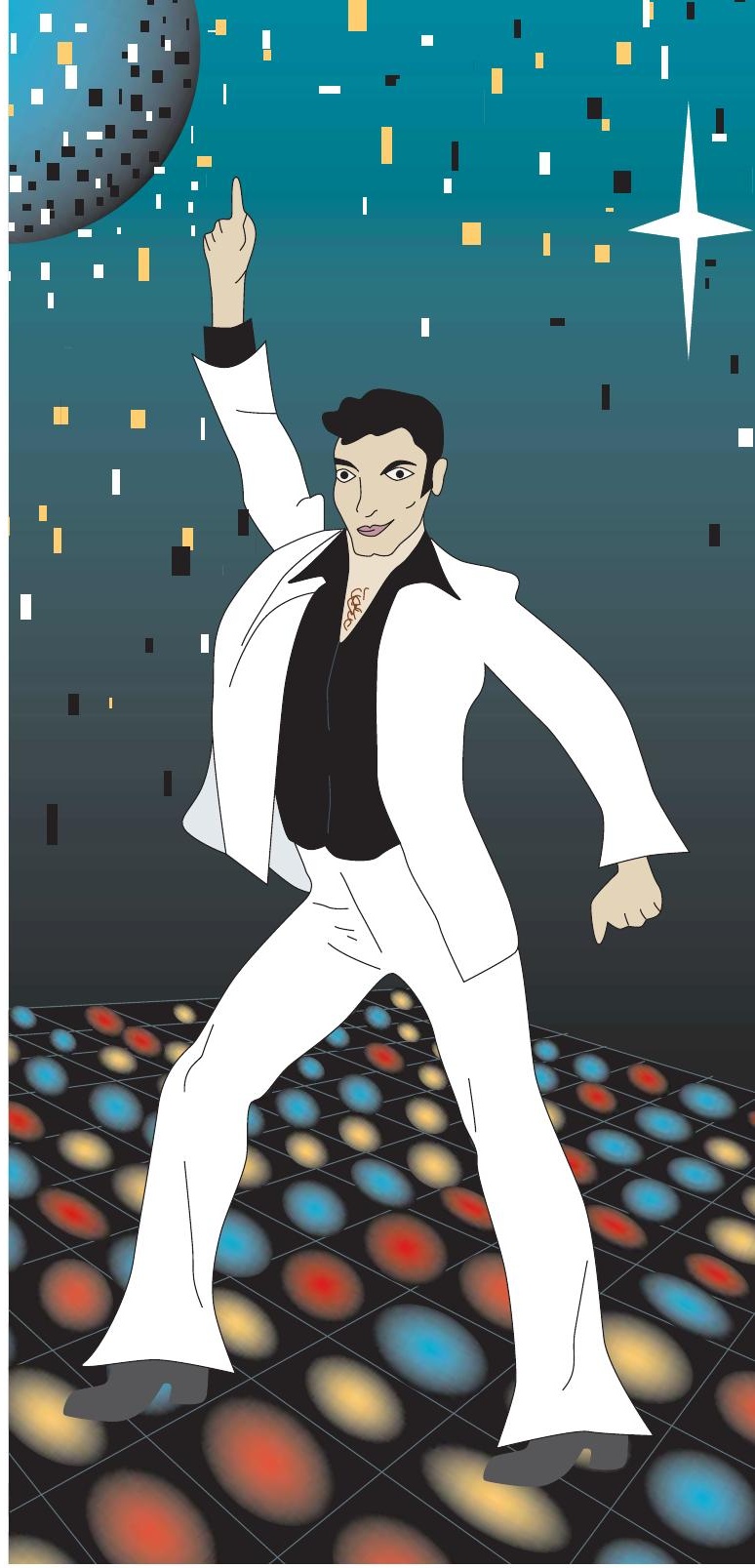

memory cells could be recovered from the $I L-7^{-/-}$ mice after one week, whereas those in control mice persisted for extended periods of time. They also showed that both central memory (lymph-node homing, $\mathrm{CD} 6 \mathrm{~L}^{+}$) and effector memory cells $\left(\mathrm{CD} 62 \mathrm{~L}^{-}\right)$, which reside in the non-lymphoid organs, require IL-7 for persistence. When memory OT-II cells were generated in OVA-immunized $I L-7^{-1-}$ hosts, cells initially proliferated, as in $I L-7 R^{-1-}$ hosts; however, OT-II cells were no longer detectable by day 21 in lymphoid or non-lymphoid organs.

Finally, both groups confirmed the requirement of IL-7 for $\mathrm{CD} 4^{+}$memory T-cell generation and persistence in recipient mice that were rendered deficient in IL-7 by antibody blocking.

Lucy Bird

(2) References and links

ORIGINAL RESEARCh PAPERS Li, J., Huston, G. \& Swain, S. L. IL-7 promotes the transition of CD4 effectors to persistent memory cells. J. Exp. Med. 15 December 2003 (doi:10.1084/jem.20030725) | Kondrack, R. M. et al. Interleukin 7 regulates the survival and generation of memory CD4 cells. J. Exp. Med. 1 December 2003 (doi:10.1084/jem.20030735) 